\title{
Cyclin $F$ is involved in response to cisplatin treatment in melanoma cell lines
}

\author{
ADRIAN KRAJEWSKI ${ }^{1}$, MACIEJ GAGAT ${ }^{1}$, AGNIESZKA ŻURYŃ $^{1}$, \\ MARTA HAŁAS-WIŚNIEWSKA ${ }^{1}$, DARIUSZ GRZANKA ${ }^{2}$ and ALINA GRZANKA ${ }^{1}$ \\ Departments of ${ }^{1}$ Histology and Embryology, and ${ }^{2}$ Clinical Pathomorphology, \\ Nicolaus Copernicus University in Toruń, Collegium Medicum in Bydgoszcz, 85-092 Bydgoszcz, Poland
}

Received May 14, 2019; Accepted October 31, 2019

DOI: $10.3892 / o r .2020 .7465$

\begin{abstract}
Cyclin F is a non-canonical cyclin which is a part of the SKP1-CUL1-F-box protein (SCF) E3 ubiquitin-protein ligase complex. Cyclin $\mathrm{F}$ is responsible for target recognition, ubiquitination, and degradation of various molecular targets. This protein also controls genome stability through the degradation of ribonucleotide reductase subunit M2 (RRM2). In the present study, the difference between cyclin F expression in cell lines derived from primary and metastatic melanoma, A375 and RPMI-7951, respectively, were investigated using a western blot analysis and flow cytometry assays. A decrease in cyclin F expression in the A375 cells and an increase in RPMI-7951 cells after cisplatin treatment were observed. These changes may be related to a mutation in p53 in the RPMI-7951 cell line. Flow cytometry was conducted to observe that the RPMI-7951 cell line exhibited greater susceptibility to cisplatin, associated with lack of proper cell cycle control. Therefore, it is possible that cyclin F may modulate drug response in melanoma. The presented data describe cyclin $\mathrm{F}$ as a new potential factor that contributes to drug resistance in melanoma patients.
\end{abstract}

\section{Introduction}

Drug resistance is a common obstacle in successfully treating cancer. Searching for new molecular targets which will increase treatment efficiency leading to key discoveries which could drive the field of oncology forward, and thus improving patient outcomes is crucial. Patients who suffer from malignant melanoma, the deadliest skin-related cancer, are still waiting for improved treatment regimen. The 5-year survival

Correspondence to: Professor Alina Grzanka, Department of Histology and Embryology, Nicolaus Copernicus University in Toruń, Collegium Medicum in Bydgoszcz, 24 Karłowicza Street, 85-092 Bydgoszcz, Poland

E-mail: agrzanka@cm.umk.pl

Key words: cyclin F, melanoma, drug resistance, cisplatin, DNA damage response of metastatic melanoma patients is still relatively low, leading to the urgent need for research in this area (1).

Cyclin $\mathrm{F}$ is a non-canonical cyclin involved in the degradation of various molecular targets through ubiquitin-mediated proteolysis. The first identified target recognized by cyclin $\mathrm{F}$ was ribonucleotide reductase subunit M2 (RRM2). The cyclin F-RRM2 axis provides a pool of DNA which can then be utilized for DNA synthesis and repair. In the G2/M phase, when DNA synthesis is complete, RRM2 is targeted for degradation. Presence of genotoxic stress induces ATR-dependent degradation of cyclin F and RRM2 can translocate to the nucleus, facilitating the accumulation of a nucleotide pool for use in DNA repair. Overexpression of RRM2 has been observed in various cancer types including lung cancer, head and neck cancer, and melanoma (2-4). Targeting RRM2 sensitizes cancer cells for drug treatment, reducing aggressiveness. The role of cyclin $\mathrm{F}$ in cancer development and treatment response, however, is still elusive. Some studies have revealed that cyclin $\mathrm{F}$ acts as a tumor suppressor, whereas other studies have revealed that cyclin $\mathrm{F}$ promotes cancer progression. To study changes in cyclin $\mathrm{F}$ following drug exposure primary and metastatic melanoma cells lines were treated with cisplatin, a compound with a well-known mechanism of action. The present study revealed that cisplatin differentially impacted cyclin $\mathrm{F}$ expression in the primary A375 melanoma cell line and the metastatic RPMI-7951 cell line. This initial study presents cyclin $\mathrm{F}$ as a new factor which may determine cellular response during drug intervention.

\section{Materials and methods}

Antibodies. The following primary antibodies were used: Cyclin F (cat. no. sc-515207; 1:100, Santa Cruz Biotechnology Inc.). RRM2 (cat. no. ab57653; 1:200, Abcam), p53 (Pab 240; cat. no. 13-4100; 1:100), p-ATR (cat. no. 720107; 1:200), p-H2. AX (cat. no. MA1-2022; 1:100), GAPDH (cat. no. MA5-15738; 1:500; all from Life Technologies; Thermo Fisher Scientific, Inc.). The following secondary antibodies were used: Alexa Fluor 594 goat anti-mouse (cat. no. A11005; 1:200), Alexa Fluor 594 donkey anti-rabbit (cat. no. A21207; 1:200), Alexa Fluor 647 anti-rabbit (cat. no. A31573; 1:500), Alexa Fluor 488 anti-mouse (cat. no. A11029; 1:500, Life Technologies; Thermo Fisher Scientific, Inc.). 
Cell culture. Two melanoma cell lines, A375 and RPMI-7951, were purchased from ATCC. The cells were cultured in DMEM (A375) or EMEM (RPMI-7951) supplemented with $10 \% \mathrm{FBS}$ and $50 \mu \mathrm{g} / \mathrm{ml}$ gentamycin and were incubated in a humidified atmosphere of $95 \%$ air $/ 5 \% \mathrm{CO}_{2}$ at $37^{\circ} \mathrm{C}$. The cell culture was tested for Mycoplasma, based on the rapid uptake of DAPI by cellular DNA. All tests were negative. All in vitro studies were performed on low passage number cells $(\mathrm{P}<5)$. The RPMI-7951 cell line bears a TP53 homozygous mutation (c.497C $>$ A) (5).

Apoptosis analysis. The presence of apoptotic cells was determined using Alexa Fluor ${ }^{\mathrm{TM}} 488$ Annexin V/Dead Cell Apoptosis Kit (Life Technologies; Thermo Fisher Scientific, Inc.) following the manufacturer's instructions. Cells were analyzed using Guava EasyCyte 6HT-2L Cytometer (Merck KGaA). FCS files were analyzed using FlowJo software (version 10.07; FlowJo LLC).

Cell cycle analysis. Cells were fixed in ethanol for $24 \mathrm{~h}$ in $-20^{\circ} \mathrm{C}$. The cells were then washed with PBS and incubated for 30 min in $\mathrm{FxCycle}^{\mathrm{TM}} \mathrm{PI} / \mathrm{RNase}$ Staining Solution (Life Technologies; Thermo Fisher Scientific, Inc). After 24 and $48 \mathrm{~h}$, cells were analyzed using Guava EasyCyte 6HT-2L Cytometer. FCS files were analyzed using InCyte software (version 3.3; Merck KGaA).

Immunofluorescence. Cells were stained using the standard protocol described in a previous study (6). Briefly, the cells were fixed with $4 \%$ paraformaldehyde blocked with $4 \%$ BSA and stained with appropriate primary and secondary antibodies. F-actin was stained using Alexa Fluor 488 phalloidin (cat. no. A12379; 1:40; Life Technologies; Thermo Fisher Scientific, Inc) (6).

Western blot assay. Whole cell lysates were prepared using RIPA buffer (Merck KGaA). Following normalization of the protein concentration, using the BCA protein assay kit (Thermo Fisher Scientific, Inc.), equal amounts of protein ( $25 \mu \mathrm{g}$ of total protein per lane) were separated using 4-12\% NuPAGE Bis-Tris Gel (Novex/Life Technologies; Thermo Fisher Scientific, Inc.) and transferred onto nitrocellulose membranes using the iBlot dry transfer system (Invitrogen; Thermo Fisher Scientific, Inc.). The membrane was processed in room temperature using iBind Flex Western Blot system (Thermo Fisher Scientific, Inc.) as described by the manufacturer. Bands were stained using 1-Step ${ }^{\mathrm{TM}}$ Ultra TMB-Blotting solution (Thermo Fisher Scientific, Inc.). Densitometry analysis was performed using ImageJ software (version 1.52q; National Institiutes of Health).

Statistical analysis. Analyses was performed using statistical software (GraphPad Prism 6; GraphPad Software, Inc.). The data were compared with the non-parametric Mann-Whitney U test or nonparametric Kruskal-Wallis test with Dunn's multiple comparisons test, and the changes were considered to indicate a statistically significant difference at a level of $\mathrm{P}<0.05$.

\section{Results}

RPMI-7951 cell line is more susceptible to cisplatin treatment. Tumor protein p53 (TP53) is a potent tumor suppressor. In the
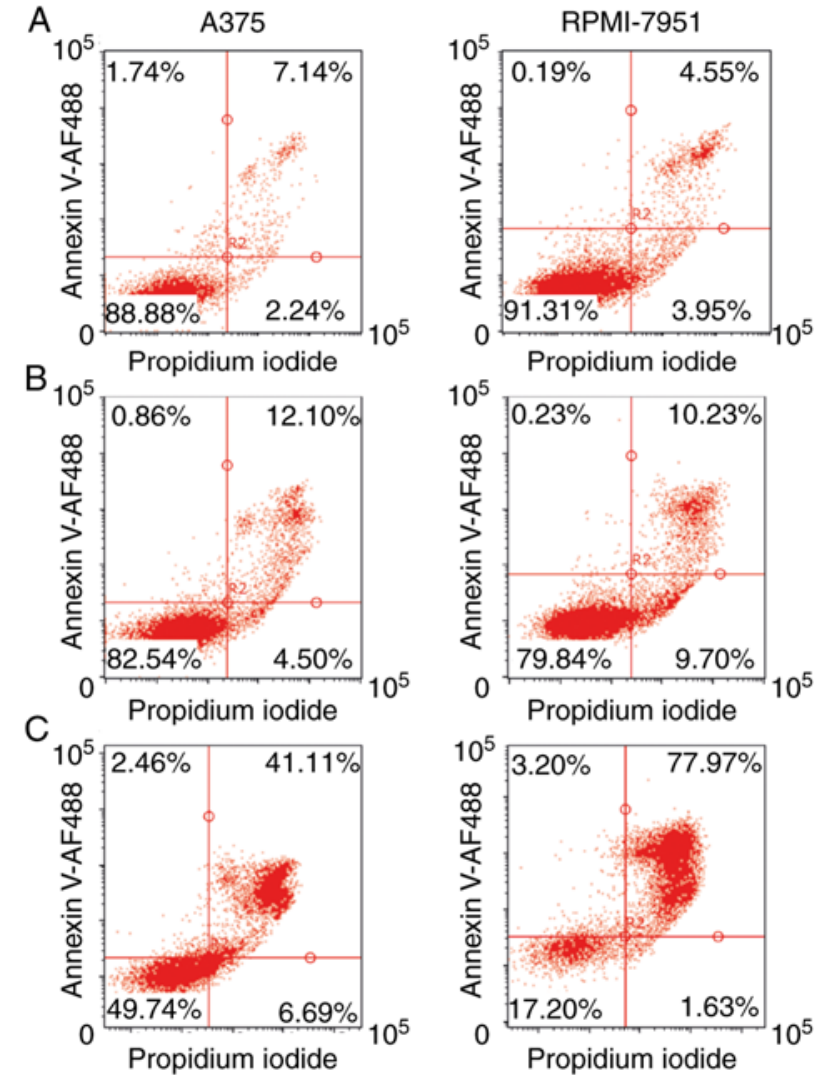

Figure 1. Apoptosis analysis of melanoma cell lines A375 and RPMI-7951. (A) Control cells. (B) Cells treated with CP for $24 \mathrm{~h}$. (C) Cells treated with $\mathrm{CP}$ for $48 \mathrm{~h}$. CP, cisplatin.

presence of DNA damage, p53 plays a dual role in the regulation of cell fate. Through the p21 pathway, p53 drives cell cycle arrest and permits the cell to repair any DNA damage (7). When the DNA damage is severe and cannot be repaired, p53 then triggers apoptosis (8). To elucidate the impact of $p 53$ on cisplatin treatment, two cell lines which differ in p53 status were selected, A375 with functional p53 and p53-mutated, RPMI-7951. After $24 \mathrm{~h}$ of cisplatin treatment, both A375 and RPMI-7951 cell lines exhibited similar, high viability with a low extent of Annexin V-positive cells. However, with prolonged, $48 \mathrm{~h}$ of treatment, the RPMI-7951 line contained a significantly higher percentage of Annexin V-positive cells compared to the A375 cell line (Fig. 1A-C). The DNA content analysis revealed that cell cycle arrest in the $\mathrm{S}$ and G2/M phase was more marked in the A375 cell line in comparison to RPMI-7951 cell line (Fig. 2A-D). An increased nuclei size corresponded with cell cycle arrest in both cell lines (Fig. 2E).

Cisplatin activates the p53 pathway in the A375 cell line. D'Angiolella et al revealed that when DNA damage occurs, cyclin $\mathrm{F}$ is downregulated, likely in an ATR-dependent manner (9). Since cisplatin induces DNA damage, p53, pATR, and $\mathrm{pH} 2 . \mathrm{AX}$ expression was analyzed. After cisplatin treatment, an increase in p-ATR and $\mathrm{p}-\mathrm{H} 2$.AX levels (Fig. 3A and B) was observed. RPMI-7951 cells bear a nonsense mutation in the p53 locus, thus an increased expression of p53 was only observed in the A375 cell line (Fig. 3C). 
A

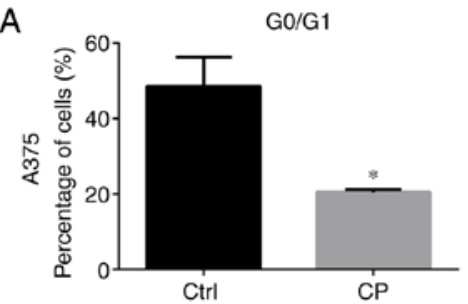

B

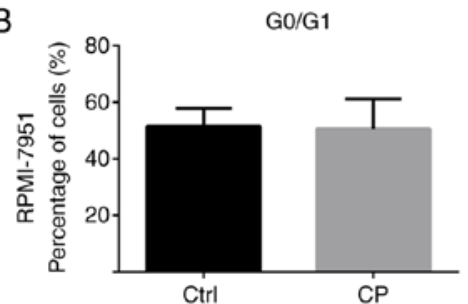

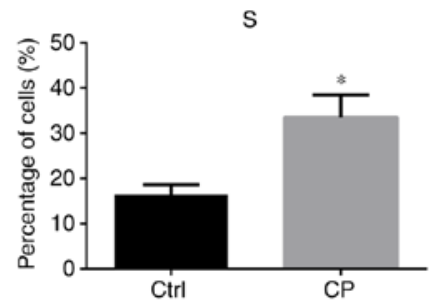

S

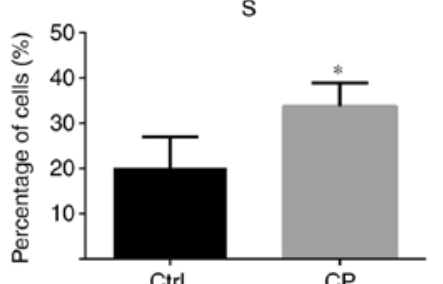

Ctrl

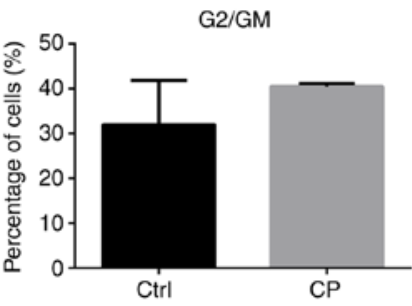

G2/GM
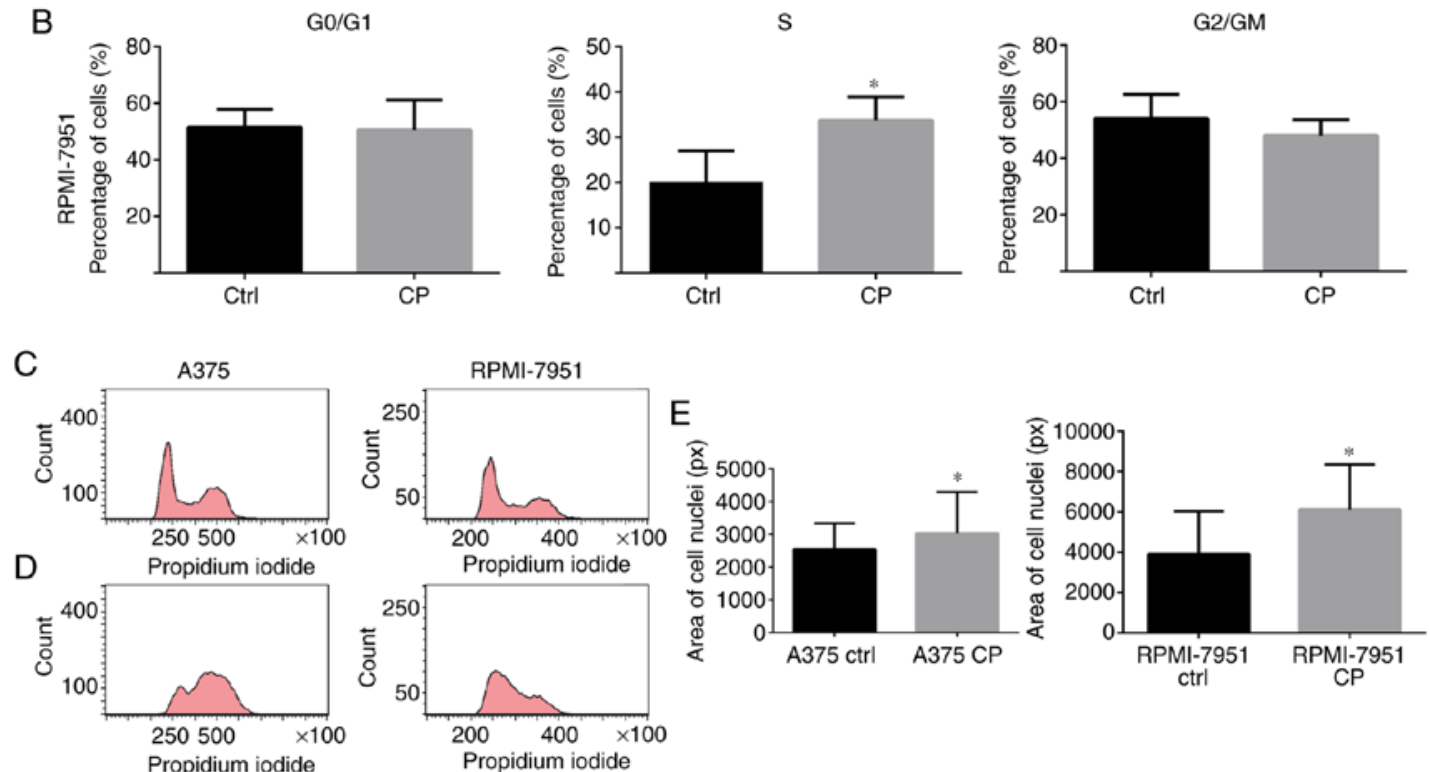

Ctrl

$\mathrm{CP}$

Figure 2. Cell cycle analysis of melanoma cell lines A375 and RPMI-7951. (A) A375 control and cells treated with CP for 24 h. (B) RPMI-7951 control and cells treated with CP for $24 \mathrm{~h}$. (C) Representative plots for the A375 and RPMI-7951 control cells. (D) Representative plots of the A549 and RPMI-7951 cells treated with CP for $24 \mathrm{~h}$ (E) Measurement of the cell nuclei area of A549 and RPMI-7951 cells after treatment with cisplatin. ${ }^{*} \mathrm{P}<0.05$. CP, cisplatin.

A

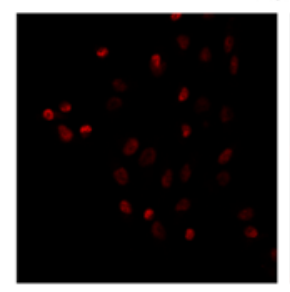

Ctrl

B

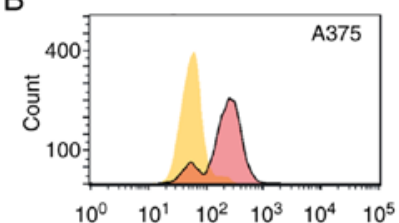

A375

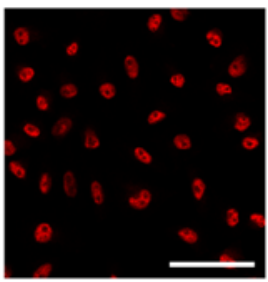

$\mathrm{CP}$

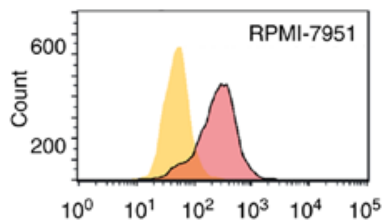

Fluorescence intensity

C

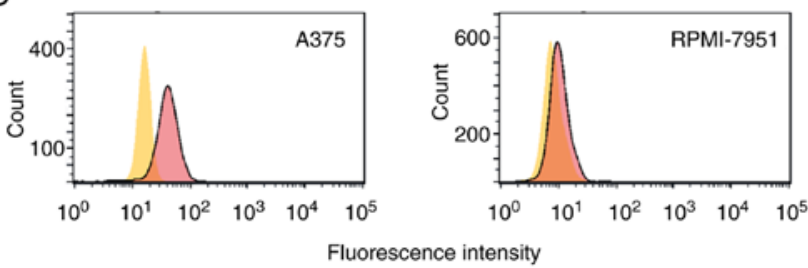

$\mathrm{CP}$
RPMI-7951

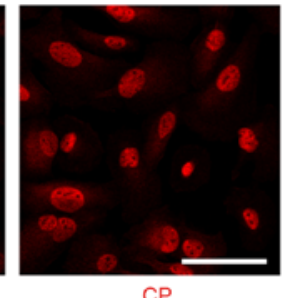

CP
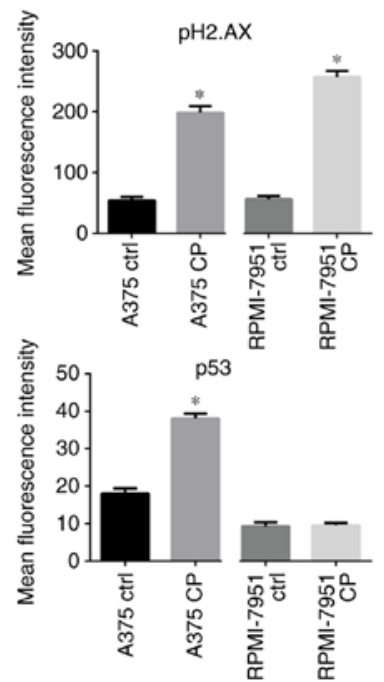

Figure 3. (A) Immunofluorescence staining of p-ATR in A375 and RPMI-7951 cell lines. (B) Flow cytometric analysis of p-H2.AX expression in A375 and RPMI-7951 cell lines. (C) Flow cytometric analysis of p53 expression in A375 and RPMI-7951 cell lines. "P<0.05 
A
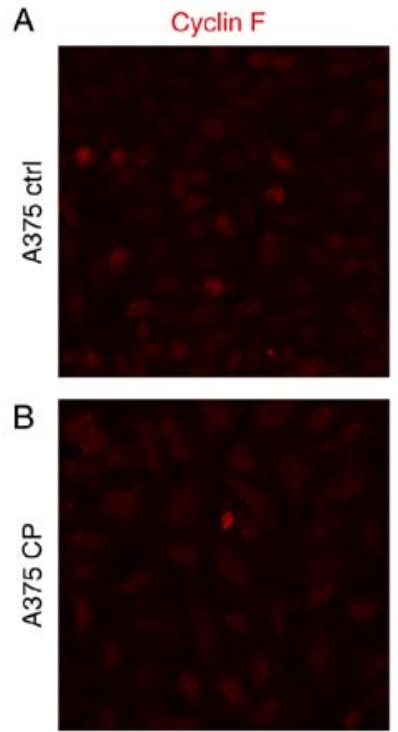

C

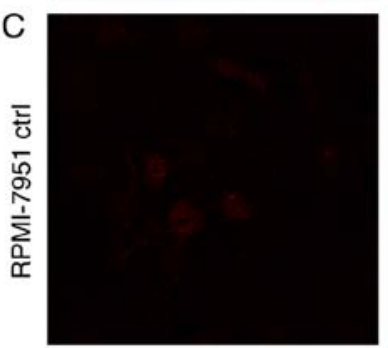

D

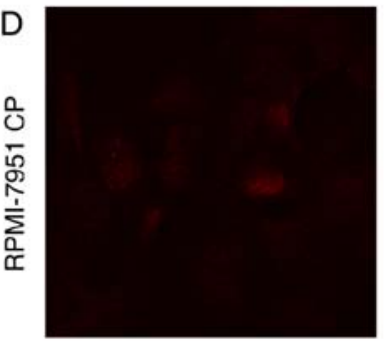

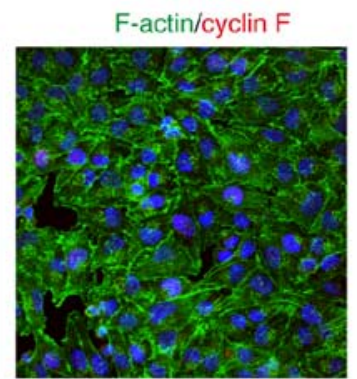
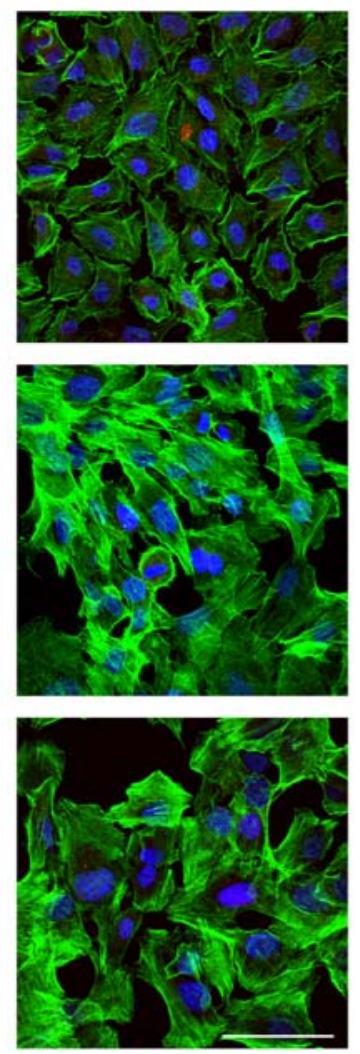

Figure 4. Confocal fluorescence microscopic examination of the localization of cyclin F in A375 and RPMI-7951 cells treated with CP. Cells were treated with $6 \mu \mathrm{M} \mathrm{CP}$ and immunolabeled for the presence of cyclin F. (A) A375 control cells. (B) A375 cells treated with CP. (C) RPMI-7951 control cells (D) RPMI-7951 cells treated with CP. Magnification, x600. CP, cisplatin.

Cisplatin downregulates cyclin F in A375 cell line but not in RPMI-7951 cells. Following cisplatin treatment, a significant decrease in cyclin F expression in the A375 cell line was observed. Immunofluorescence staining revealed that the percentage of treated cells with a strong nuclear cyclin F signal was significantly lower than observed in the control cells (Fig. 4). Notably, this effect was not observed in the RPMI-7951 cell line. Flow cytometric analysis revealed a significant increase in mean fluorescence intensity in the RPMI-7951 cell line after the cisplatin treatment compared to the control group (Fig. 5A and C). While both cell lines exhibited nuclear localization of cyclin $\mathrm{F}$, with weak but positive cytoplasmic staining, this was more marked in the RPMI-7951 cell line (Fig. 6). Western blot analysis confirmed the aforementioned observations (Fig. 5D).

Cisplatin upregulates the RRM2 expression. Cisplatin is a well-characterized DNA damage-inducer and it is well-known

that alterations in the DNA repair pathway can drive resistance to DNA-focused agents. RRM2 plays a central role in the synthesis of deoxyribonucleotides from ribonucleotides (10). Overexpression of RRM2 has been associated with drug resistance and worse prognosis for cancer patients (11). The degradation of RRM2 is regulated by cyclin F, a functional axis which controls genome integrity. To investigate how the p53 status impacts RRM2 flow cytometry was performed and western blot assays to assess the level of RRM2 in the A375 and RPMI-7951 cell lines. After the treatment with cisplatin, western blot analysis revealed an increased expression of RRM2 in both melanoma cell lines compared to the controls (Fig. 5E). Moreover, flow cytometry was also conducted to assess the mean fluorescence intensity of the RRM2 protein. The flow cytometric data revealed a markedly higher increase of RRM2 in the RPMI-7951 cell line relative to the A375 cell line (Fig. 5B and C). The immunofluorescence staining revealed a shift of RRM2 from cytoplasmic to cellular localization (Fig. 6).

\section{Discussion}

Understanding the molecular mechanisms responsible for cancer development, metastasis and migration of tumor cells, as well as cancer aggressiveness are crucial in designing improved treatment strategies to benefit cancer patients. Drug resistance is a significant obstacle in achieving satisfactory effects of therapy. D'Angiolella et al described the functional axis which comprises genome stability and undisturbed cell proliferation as associated with cyclin F (9). Cyclin F regulates the pool of nucleotides available for DNA synthesis and repair, through proteasome-mediated degradation of RRM2. The overexpression of RRM2 is common in multiple cancer types including lung, head and neck, and gastric cancer. Furthermore, patients with high RRM2 expression are characterized with a worse prognosis (12). While the role of RRM2 in cancer development and progression is well-established, little is known about how changes in cyclin F expression may affect cancer development and drug response. Fu et al revealed that low cyclin $\mathrm{F}$ expression is associated with a worse prognosis for hepatocarcinoma patients. Downregulation of cyclin F was correlated with tumor size, differentiation, clinical stage, and tumor multiplicity (13). Conversely, in glioma cells, cyclin F was reported as a tumor-suppressive factor. A study by Deshmukh et al revealed that gliomas are characterized by lower cyclin $\mathrm{F}$ expression in comparison with normal brain tissue. Moreover, depletion of cyclin F using shRNA resulted in increased tumor size and formation of numerous metastatic nodules in the lungs and liver. Additionally, a decrease in cyclin $\mathrm{F}$ expression coincided with increased circulating tumor cells, affected epithelial markers including E-cadherin, and increased expression of mesenchymal markers such as vimentin and fibronectin (14). On the other hand, cyclin F has been reported as an oncogene in ovarian cancer. Through the OCT4-Nipp1/Ccnf-PP1-pRb axis, cyclin F was involved with the phosphorylation of retinoblastoma protein. Activation of the pathway resulted in enhanced tumor proliferation and increased expression of the chromosomal passenger complex (CPC) elements such as Aurora B, survivin and borealin. The present study indicated that treatment of melanoma cells with 

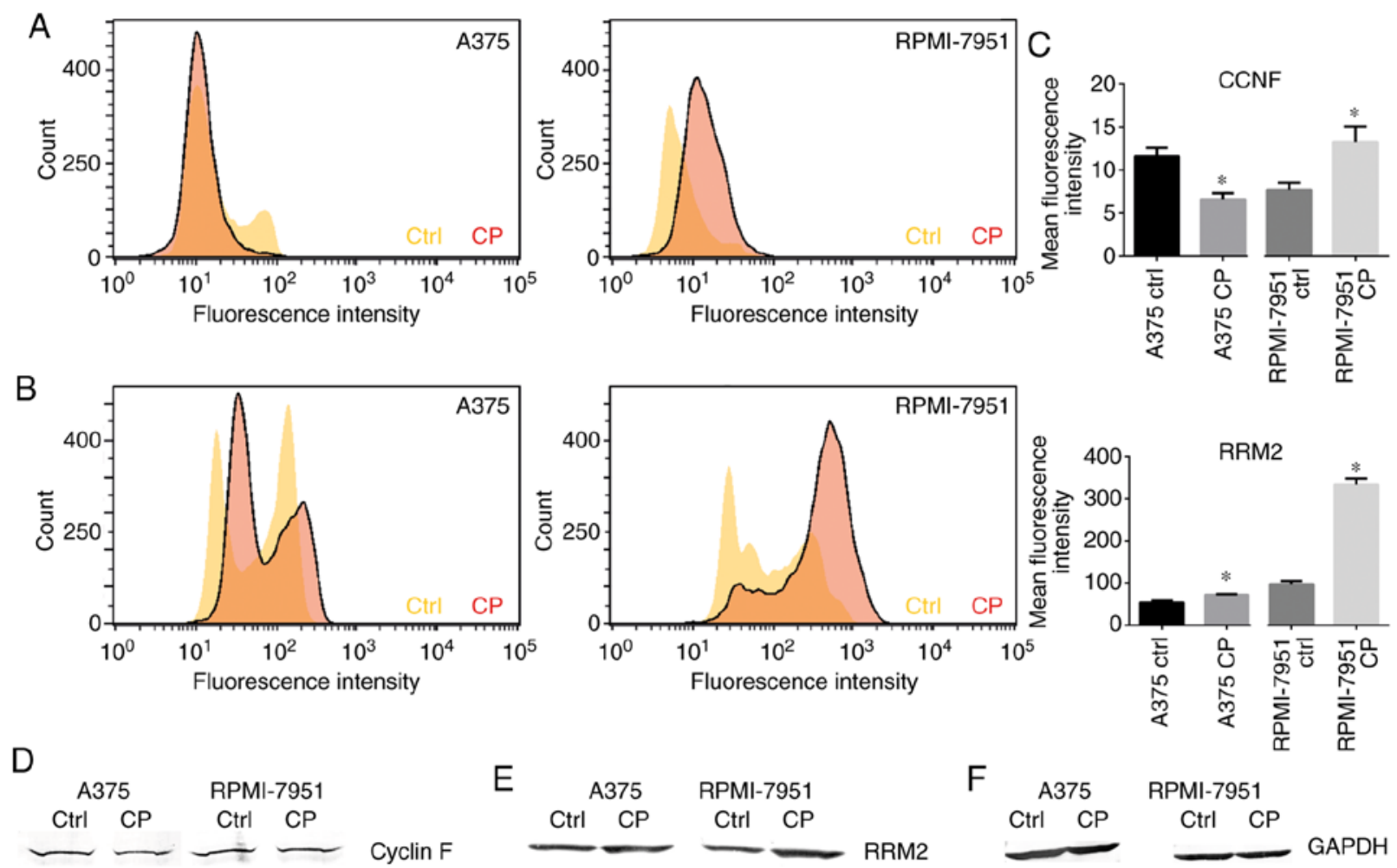

Figure 5. Flow cytometric analysis of cyclin F and RRM2 expression. Flow cytometric analysis of (A) cyclin F expression and (B) RRM2 expression. (C) ${ }^{*} \mathrm{P}<0.05$, statistically significant difference between the control and treated sample (Kruskal-Wallis with Dunn's post hoc test). Western blot analysis of (D) cyclin F, (E) RRM2 and (F) loading control (GAPDH) expression. CP, cisplatin.

cisplatin resulted in a greater decrease in cyclin $\mathrm{F}$ expression in A375 compared to the p53-mutant RPMI-7951 cell line. These results were consistent with the work of D'Angiolella et al (9) revealing downregulation of cyclin $\mathrm{F}$ after cisplatin treatment. The abundance of functional p53 results in ineffective cyclin $\mathrm{F}$ elimination and increased apoptosis due to the inability of cells to undergo cell cycle arrest. These findings support the notion that cyclin $\mathrm{F}$ acts as a tumor suppressor. However, the exact mechanism of the oncogenic properties of this protein remain unclear. Cyclin F is reported to provide genome stability through ubiquitin-mediated proteolysis of CP110, NUSAP, and RRM2. Enrichment of the CP110 protein leads to overduplication of the centrosome and mitotic aberrations. CP110 is stabilized by the USP33 protein, which de-ubiquitinates and prevents degradation via cyclin F-mediated proteolysis. Centrosome amplification is a common event in melanoma cells (15). It has been proposed that most of the amplified centrosomes are a result of centriole overduplication (16). The downregulation of cyclin F can contribute to genome instability and development of cells with malignant properties.

The faithful replication of DNA cannot be conducted without an appropriate pool of nucleotides delivered in proper time. RRM2 is a functional part of the ribonucleotidase reductase enzyme. During cell cycle progression, the activity of RRM2 increases and peaks at the $\mathrm{S}$ phase, when demand for the nucleotides used in DNA synthesis is the highest. During the G2/M phase, RRM2 is phosphorylated and directed to proteasome-mediated degradation. This degradation of RRM2 is regulated by cyclin F (9). While cisplatin is not a cell cycle-specific drug, cells are the most susceptible for treatment during the G1 phase. Cisplatin alternates the expression of cell-cycle related genes and creates subsequent cell cycle arrest in the G2 phase (17). Cisplatin induces the p53 protein and functions as a p $21^{\mathrm{CIP} / \mathrm{WAF} 1}$ inhibitor, which stops cell cycle progression. It has been demonstrated that mutation in the p53 gene may significantly increase sensitivity, while the accumulation of p53 is partially responsible for cisplatin resistance $(18,19)$. The efficient repair of DNA damage after the genotoxic stress via RRM2 requires downregulation of cyclin F. In the present study, higher RRM2 fluorescence intensity after cisplatin treatment was observed in the RPMI-7951, mutant p53 cell line. The basal level of cyclin $F$ in the RPMI-7951 cell line was slightly higher compared to control cells, indicating that the degradation rate of RRM2 should also be greater. However, this phenomenon did not occur. Since the degradation of RRM2 is p53-dependent, p53-mutated RPMI-7951 cells accumulate RRM2, even in the presence of cyclin F. The lack of functional p53 prevents cell cycle arrest, leading to the high pools of nucleotides which cannot be utilized for DNA repair. Increased susceptibility for cisplatin treatment in the RPMI-7951 p53-mutated metastatic melanoma cell line compared to the p53 wild-type A375 primary melanoma cell line was observed. The inhibition of RRM2 by 553 follows suppression of mammalian target of rapamycin complex 1 (mTORC1) (20). Moreover, mutations in p53 have been revealed to cause increased levels of RRM1 and RRM2 in various cancer cell lines $(20,21)$. This data indicated the paramount role of p53 in the regulation of RRM2 expression. Effective DNA damage repair depends on the ability of the cell to pause cell cycle progression through the activation of the 
A
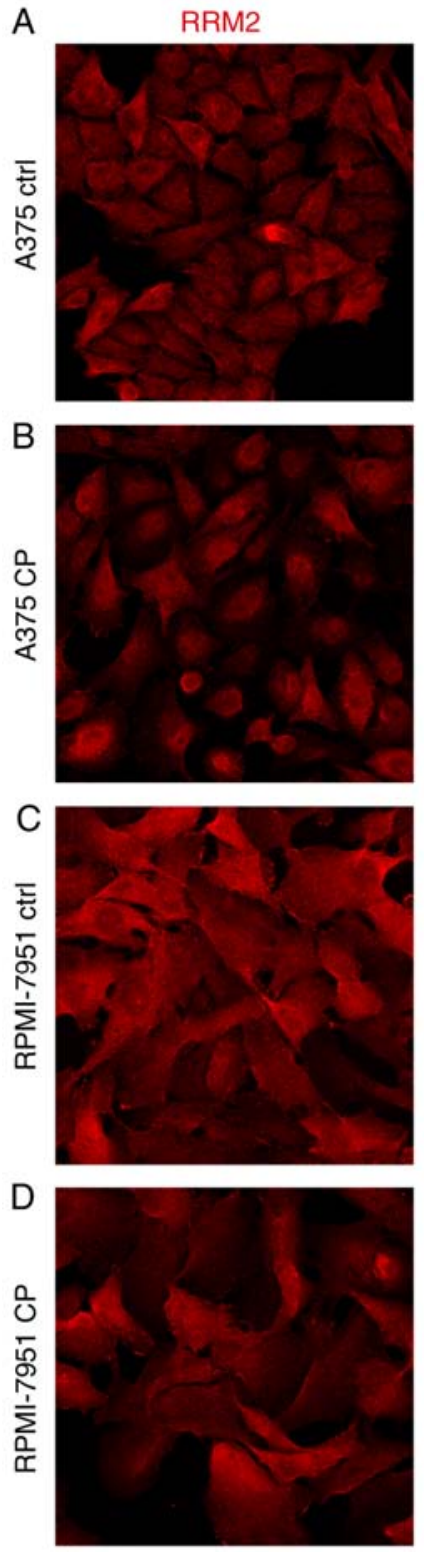

Figure 6. Confocal fluorescence microscopic examination of the localization of RRM2 in A375 and RPMI-7951 cells treated with CP. Cells were treated with $6 \mu \mathrm{M} \mathrm{CP}$ and immunolabeled for the presence of RRM2. (A) A375 control cells. (B) A375 cells treated with CP. (C) RPMI-7951 control cells. (D) RPMI-7951 cells treated with CP. Magnification, x600. CP, cisplatin.

cell cycle checkpoints. If the cells enter the next cycle phase with unrepaired DNA, this may trigger unfaithful DNA replication or improper cell division, leading to cancer. When cells are exposed to ionizing radiation (IR), cyclin F promotes entry into a cell cycle checkpoint, suppressing the oncogenic B-Myb protein. The interaction between cyclin $\mathrm{F}$ and $\mathrm{B}-\mathrm{Myb}$ prevents cyclin A-mediated phosphorylation of B-Myb and suppresses its activity, which is necessary for recovery from cell cycle arrest after genotoxic stress (22). Moreover, the degradation of cyclin $\mathrm{F}$ via $\beta$-TrCP is required for $\mathrm{G} 2 / \mathrm{M}$ transition and activates the transcription of the mitosis-related enzymatic machinery (23).

The present observations support the role of cyclin $\mathrm{F}$ as a tumor suppressor in melanoma. However, there are several studies which indicate the potential oncogenic effects of cyclin F. Cancer stem-like cells are identified in many tumor types. The presence of stem-like cells confers to increased tumor-initiating potential, chemoresistance, apoptosis resistance, and enhanced EMT-associated events (24-26). Oct-4 and Nanog are important transcription factors, essential for the self-renewal of embryonic stem cells (27). It has been reported that the expression of Oct-4 and Nanog in several cancers increases malignancy and is associated with poor prognosis. It has also been revealed that Oct-4 expression is a strong prognostic marker which can be utilized to predict poor clinicopathological and prognostic characteristics in non-small cell lung cancer (28). Oct-4 has been revealed to drive Nanog and cyclin F expression, both inhibitors of protein phosphatase 1 (PP1), preventing the dephosphorylation of $\mathrm{Rb}$ and increasing the cell proliferation rate (29). It is possible that cyclin $\mathrm{F}$, under specific circumstances, can act as an oncogene rather than a tumor suppressor gene. Activation of Oct4/Nanog signaling was revealed to enhance stem-like properties such as spindle shape, foci formation, and increased levels of CD133. A549 cells overexpressing OCT4 achieved the ability to form spheres in suspension and were characterized by higher resistance to cisplatin (30). Oct4 overexpression was also revealed to contribute to gefitinib resistance and increased self-renewal capacity in PC9 and HCC827 cell lines (28). However, strong OCT4 expression in the A375 or RPMI-7951 cell lines was not observed (data not shown). Other investigators have indicated a crucial role of Oct-4 in carcinogenesis and metastasis events in malignant melanoma. Expression of Nanog and Oct-4 markers were revealed to be associated with higher tumor aggressiveness and invasiveness. Oct-4 overexpression induced ameboid migration markers and increased extravasation and transmigration capacities in melanoma cell lines (31).

It has been revealed that A549 non-small cell lung cancer cells grown on 3D scaffolds were characterized by higher cyclin $\mathrm{F}$ expression and were more radio-resistance compared to 2D cultured cells (32). Hepatocellular carcinoma HepG2 cells treated with polysaccharides from abalone had stimulatory potential and exhibited increased mRNA levels of cyclin B, CDK1, and cyclin F and reduced levels of cyclin E and CDK6 (33). It is possible that the expression profile of cyclin $\mathrm{F}$ and its oncogenic or tumor suppressor effect depends on the cellular state and cannot be described with simple relationships. Choudhury et al revealed that cyclin $\mathrm{F}$ is a substrate for oncogenic kinase AKT1. The phosphorylation of cyclin $\mathrm{F}$ resulted in increased stability and promoted the G1/S transition. Stabilization of cyclin F promoted Cdh1 degradation, allowing S-phase entry. Cyclin F may act as an oncogene promoting the degradation of Cdh1 and AKT1 activation $(34,35)$. Conversely, at the end of the $S$ phase, cyclin $\mathrm{F}$ takes part in Cdc6 degradation, preventing DNA re-replication.The absence of cyclin $\mathrm{F}$ provoked genome instability and allowed more than one replication event per cell cycle (36). Additionally, cyclin F was revealed to target SLBP for proteasome-mediated degradation in the G2 phase. In the presence of SLBP, G2 phase translation of H2A.X histone mRNA increased. Elevated H2A.X levels promoted apoptosis upon the genotoxic stress. The degradation of SLBP via cyclin $\mathrm{F}$ led to increased cell proliferation and decreased cytotoxicity of agents. These data revealed the potential role 
of cyclin $\mathrm{F}$ in drug response (37). All the presented data indicate a dual role of cyclin $\mathrm{F}$ in integrating cell cycle control and maintaining genome stability. Few studies on cancer cells have revealed that low cyclin $\mathrm{F}$ expression is associated with worse prognosis and increased proliferation of the cells. Thus, is important to define the circumstances when cyclin $\mathrm{F}$ bears oncogenic properties. Our previous research analyzed The Cancer Genome Atlas data and revealed that high expression of cyclin F mRNA was associated with poor prognosis and increased activity of pathways related to the cell cycle and DNA damage repair (38).

In conclusion, it was demonstrated that cyclin $\mathrm{F}$ was involved in the response of melanoma cell lines to the cisplatin treatment. The change in cyclin $\mathrm{F}$ expression in response to cisplatin treatment was significantly different in A375, a primary melanoma cell line, and RPMI-7951, a metastatic melanoma cell line. The observed difference may be related to the p53 mutation in the RPMI-7951 cell line, which results in increased levels of cyclin $\mathrm{F}$ and a simultaneous increase in RRM2 (5). Further investigations must be conducted to elucidate the role of cyclin $\mathrm{F}$ in drug response and regulation of the tumor invasiveness.

\section{Acknowledgements}

Not applicable.

\section{Funding}

The present study was supported by a grant from the National Science Centre, Poland (grant no. 2016/21/B/NZ7/01121 to AG).

\section{Availability of data and materials}

The datasets used during the present study are available from the corresponding author upon reasonable request.

\section{Authors' contributions}

AK and AG conceived and designed the study. AK, MG, AZ and $\mathrm{MHW}$ performed the experiments and $\mathrm{AK}$ and $\mathrm{MG}$ wrote the manuscript. DG performed the statistical analysis. AK and MHW reviewed the manuscript and AG supervised the project. All authors read and approved the manuscript and agree to be accountable for all aspects of the research in ensuring that the accuracy or integrity of any part of the work are appropriately investigated and resolved.

\section{Ethics approval and consent to participate}

Not applicable.

\section{Patient consent for publication}

Not applicable.

\section{Competing interests}

The authors declare that they have no competing interests.

\section{References}

1. Maverakis E, Cornelius LA, Bowen GM, Phan T, Patel FB, Fitzmaurice S, He Y, Burrall B, Duong C, Kloxin AM, et al: Metastatic melanoma-a review of current and future treatment options. Acta Dermato Venereol 95: 516-524, 2015.

2. Fatkhutdinov N, Sproesser K, Krepler C, Liu Q, Brafford PA, Herlyn M, Aird KM and Zhang R: Targeting RRM2 and mutant BRAF is a novel combinatorial strategy for melanoma. Mol Cancer Res 14: 767-775, 2016.

3. Rahman MA, Amin AR, Wang D, Koenig L, Nannapaneni S, Chen Z, Wang Z, Sica G, Deng X, Chen ZG and Shin DM: RRM2 regulates Bcl-2 in head and neck and lung cancers: A potential target for cancer therapy. Clin Cancer Res 19: 3416-3428, 2013.

4. Wang L, Meng L, Wang XW, Ma GY and Chen JH: Expression of RRM1 and RRM2 as a novel prognostic marker in advanced non-small cell lung cancer receiving chemotherapy. Tumor Biol 35: 1899-1906, 2014.

5. Bamford S, Dawson E, Forbes S, Clements J, Pettett R, Dogan A, Flanagan A, Teague J, Futreal PA, Stratton MR and Wooster R: The COSMIC (catalogue of somatic mutations in cancer) database and website. Br J Cancer 91: 355-358, 2004.

6. Żuryń A, Krajewski A, Klimaszewska-Wiśniewska A, Grzanka A and Grzanka D: Expression of cyclin B1, D1 and K in non-small cell lung cancer H1299 cells following treatment with sulforaphane. Oncol Rep 41: 1313-1323, 2019.

7. Levine AJ: p53, the cellular gatekeeper for growth and division. Cell 88: 323-331, 1997.

8. Lowe SW, Ruley HE, Jacks T and Housman DE: p53-dependent apoptosis modulates the cytotoxicity of anticancer agents. Cell 74: 957-967, 1993.

9. D'Angiolella V, Donato V, Forrester FM, Jeong YT, Pellacani C, Kudo Y, Saraf A, Florens L, Washburn MP and Pagano M: Cyclin F-mediated degradation of ribonucleotide reductase M2 controls genome integrity and DNA repair. Cell 149: 1023-1034, 2012.

10. Shao J, Zhou B, Chu B and Yen Y: Ribonucleotide reductase inhibitors and future drug design. Curr Cancer Drug Targets 6: 409-431, 2006

11. Su YF, Wu TF, Ko JL, Tsai HT, Tee YT, Chien MH, Chou CH, Lin WL, Low HY, Chou MY, et al: The expression of ribonucleotide reductase M2 in the carcinogenesis of uterine cervix and its relationship with clinicopathological characteristics and prognosis of cancer patients. PLoS One 9: e91644, 2014.

12. Zhong Z, Cao Y, Yang S and Zhang S: Overexpression of RRM2 in gastric cancer cell promotes their invasiveness via AKT/ NF- $\kappa$ B signaling pathway. Pharmazie 71: 280-284, 2016.

13. Fu J, Qiu H, Cai M, Pan Y, Cao Y, Liu L, Yun J and Zhang CZ: Low cyclin $\mathrm{F}$ expression in hepatocellular carcinoma associates with poor differentiation and unfavorable prognosis. Cancer Sci 104: 508-515, 2013.

14. Deshmukh RS, Sharma S and Das S: Cyclin F-dependent degradation of RBPJ inhibits IDH1 ${ }^{132} \mathrm{H}_{\text {-mediated tumorigenesis. }}$ Cancer Res 78: 6386-6398, 2018.

15. Li J, D'Angiolella V, Seeley ES, Kim S, Kobayashi T, Fu W, Campos EI, Pagano M and Dynlacht BD: USP33 regulates centrosome biogenesis via deubiquitination of the centriolar protein CP110. Nature 495: 255-259, 2013.

16. Denu RA, Shabbir M, Nihal M, Singh CK, Longley BJ, Burkard ME and Ahmad N: Centriole overduplication is the predominant mechanism leading to centrosome amplification in melanoma. Mol Cancer Res 16: 517-527, 2018.

17. Donaldson KL, Goolsby GL and Wahl AF: Cytotoxicity of the anticancer agents cisplatin and taxol during cell proliferation and the cell cycle. Int J Cancer 57: 847-855, 1994.

18. Perego P, Giarola M, Righetti SC, Supino R, Caserini C, Delia D, Pierotti MA, Miyashita T, Reed JC and Zunino F: Association between cisplatin resistance and mutation of p53 gene and reduced bax expression in ovarian carcinoma cell systems. Cancer Res 56: 556-562, 1996.

19. Hawkins DS, Demers GW and Galloway DA: Inactivation of p53 enhances sensitivity to multiple chemotherapeutic agents. Cancer Res 56: 892-898, 1996.

20. He Z, Hu X, Liu W, Dorrance A, Garzon R, Houghton PJ and Shen C: P53 suppresses ribonucleotide reductase via inhibiting mTORC1. Oncotarget 8: 41422-41431, 2017.

21. Kollareddy M, Dimitrova E, Vallabhaneni KC, Chan A, Le T, Chauhan KM, Carrero ZI, Ramakrishnan G, Watabe K, Haupt Y, et al: Regulation of nucleotide metabolism by mutant $\mathrm{p} 53$ contributes to its gain-of-function activities. Nat Commun 6: 7389, 2015. 
22. Klein DK, Hoffmann S, Ahlskog JK, O'Hanlon K, Quaas M Larsen BD, Rolland B, Rösner HI, Walter D, Kousholt AN, et al: Cyclin F suppresses B-Myb activity to promote cell cycle checkpoint control. Nat Commun 6: 5800, 2015.

23. Mavrommati I, Faedda R, Galasso G, Li J, Burdova K, Fischer R, Kessler BM, Carrero ZI, Guardavaccaro D, Pagano $M$ and D'Angiolella V: $\beta$-TrCP- and casein kinase II-mediated degradation of cyclin $\mathrm{F}$ controls timely mitotic progression. Cell Rep 24: 3404-3412, 2018.

24. Chen T, You Y, Jiang H and Wang ZZ: Epithelial-mesenchymal transition (EMT): A biological process in the development, stem cell differentiation, and tumorigenesis. J Cell Physiol 232: 3261-3272, 2017.

25. Phi LTH, Sari IN, Yang YG, Lee SH, Jun N, Kim KS, Lee YK and Kwon HY: Cancer stem cells (CSCs) in drug resistance and their therapeutic implications in cancer treatment. Stem Cells Int 2018: 5416923, 2018.

26. Xu Y, So C, Lam HM, Fung MC and Tsang SY: Apoptosis reversal promotes cancer stem cell-like cell formation. Neoplasia 20: 295-303, 2018

27. Loh YH, Wu Q, Chew JL, Vega VB, Zhang W, Chen X, Bourque G, George J, Leong B, Liu J, et al: The Oct4 and Nanog transcription network regulates pluripotency in mouse embryonic stem cells. Nat Genet 38: 431-440, 2006.

28. Li SJ, Huang J, Zhou XD, Zhang WB, Lai YT and Che GW: Clinicopathological and prognostic significance of Oct-4 expression in patients with non-small cell lung cancer: A systematic review and meta-analysis. J Thorac Dis 8: 1587-1600, 2016.

29. Comisso E, Scarola M, Rosso M, Piazza S, Marzinotto S, Ciani Y, Orsaria M, Mariuzzi L, Schneider C, Schoeftner S and Benetti R: OCT4 controls mitotic stability and inactivates the RB tumor suppressor pathway to enhance ovarian cancer aggressiveness. Oncogene 36: 4253-4266, 2017.

30. Chiou SH, Wang ML, Chou YT, Chen CJ, Hong CF, Hsieh WJ, Chang HT, Chen YS, Lin TW, Hsu HS and Wu CW: Coexpression of Oct4 and nanog enhances malignancy in lung adenocarcinoma by inducing cancer stem cell-like properties and epithelial-mesenchymal transdifferentiation. Cancer Res 70 10433-10444, 2010
31. Borrull A, Ghislin S, Deshayes F, Lauriol J, Alcaide-Loridan C and Middendorp S: Nanog and Oct4 overexpression increases motility and transmigration of melanoma cells. J Cancer Res Clin Oncol 138: 1145-1154, 2012

32. Pan D, Chen Y, Du Y, Ren Z, Li X and Hu B: Methylation of promoter of RBL1 enhances the radioresistance of three dimensional cultured carcinoma cells. Oncotarget 8: 4422-4435, 2017.

33. Wang YM, Wu FJ, Du L, Li GY, Takahashi K, Xue Y and Xue CH: Effects of polysaccharides from abalone (Haliotis discus hannai Ino) on HepG2 cell proliferation. Int J Biol Macromol 66: 354-361, 2014.

34. Choudhury R, Bonacci T, Wang X, Truong A, Arceci A, Zhang Y, Mills CA, Kernan JL, Liu P and Emanuele MJ: The E3 ubiquitin ligase $\mathrm{SCF}(\mathrm{Cyclin} \mathrm{F})$ transmits AKT signaling to the cell-cycle machinery. Cell Rep 20: 3212-3222, 2017.

35. Choudhury R, Bonacci T, Arceci A, Lahiri D, Mills CA, Kernan JL, Branigan TB, DeCaprio JA, Burke DJ and Emanuele MJ: APC/C and SCF (cyclin F) constitute a reciprocal feedback circuit controlling s-phase entry. Cell Rep 16: 3359-3372, 2016.

36. Walter D, Hoffmann S, Komseli ES, Rappsilber J, Gorgoulis V and Sørensen CS: SCF(Cyclin F)-dependent degradation of CDC6 suppresses DNA re-replication. Nat Commun 7: 10530, 2016.

37. Dankert JF, Rona G, Clijsters L, Geter P, Skaar JR, Bermudez-Hernandez K, Sassani E, Fenyö D, Ueberheide B, Schneider R and Pagano M: Cyclin F-mediated degradation of SLBP limits H2A.X accumulation and apoptosis upon genotoxic stress in G2. Mol Cell 64: 507-519, 2016

38. Gagat M, Krajewski A, Grzanka D and Grzanka A: Potential role of cyclin $\mathrm{F}$ mRNA expression in the survival of skin melanoma patients: Comprehensive analysis of the pathways altered due to cyclin F upregulation. Oncol Rep 40: 123-144, 2018.

This work is licensed under a Creative Commons Attribution-NonCommercial-NoDerivatives 4.0 International (CC BY-NC-ND 4.0) License. 